\title{
THE SUN'S ROTATION RATE AS INFERRED FROM MAGNETIC FIELD DATA
}

\author{
J.O. STENFLO \\ Institute of Astronomy \\ ETH-Zentrum, CH-8092 Zürich \\ Switzerland
}

\begin{abstract}
The pattern of solar magnetic fields has been used as a tracer to determine how the sun's rotation rate varies with latitude and time. Two distinctly different rotation laws emerge from such an analysis, one agreeing with the surface Doppler rotation rate, the other corresponding to much more rigid rotation with a small polar spin-up. Detailed analysis shows that this second law cannot be explained in terms of flux redistribution on the solar surface, but that it represents the rotation properties of the sources of magnetic flux, which are likely to be located at the bottom of the convection zone.

The rotational phase velocity of the source pattern is found to be constant with time, which suggests that the depth at which the magnetic flux is stored and amplified inside the sun does not vary with the solar cycle, and that the phase velocity also represents the plasma velocity.
\end{abstract}

\section{The Two Differential Rotation Laws for the Magnetic Field Pattern}

We have long been used to the "peaceful coexistence" of various rotation laws on the sun: The determined angular velocity of rotation and its variation with latitude is different for different tracers used, like sunspots, prominences, or coronal holes, and also differs from the rotation rate determined from the Doppler shifts of spectral lines (cf. Van Tend and Zwaan, 1976). It is surprising, however, that the pattern phase velocity of photospheric magnetic fields is also found to be greatly different when different types of correlation analyses are carried out.

This is illustrated in Figure 1. The solid line shows the synodic rotation period determined by Snodgrass (1983) from a cross-correlation analysis of daily Mt Wilson magnetograms. It agrees well with the Doppler rate (dashed-dotted line) of Howard et al. (1983). In striking contrast is the pattern rotation rate determined from an autocorrelation analysis of $\mathrm{Mt}$ Wilson - Kitt Peak synoptic magnetic field data over a 26 yr period (Stenflo, 1989). It is given by the four types of symbols in Figure 1, which refer to the four different autocorrelation peaks (with lags from 1 to 4 solar rotation periods) used. While the Snodgrass (1983) rotation law exhibits a steep latitude dependence, with a rotation period of about 38 days near the poles, the autocorrelation results give a rotation period that reaches a maximum of 29-30 days at a latitude of $50-55^{\circ}$, with a tendency for a small polar spin-up.

This huge discrepancy between a quasi-rigid and a steep differential rotation cannot be 


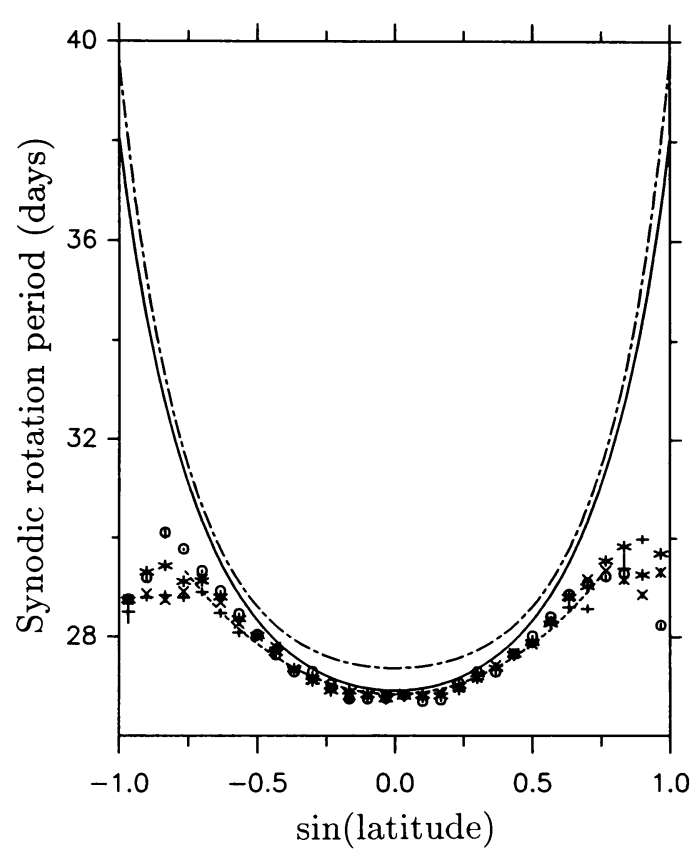

Fig. 1. Differential rotation of the sun's magnetic field pattern. The symbols (circles, stars, pluses, crosses) represent the autocorrelation results of Stenflo (1989), indicating a quasi-rigid rotation law with a small polar spin-up. The solid curve represents the cross-correlation results of Snodgrass (1983), which are in good agreement with the Doppler results (dashed-dotted curve), from Howard et al. (1983).

explained in terms of any statistical errors or artifacts. It also cannot be explained by variations with time of the solar rotation, since a detailed analysis (see below) shows that the quasi-rigid rotation law is time invariant over the $26 \mathrm{yr}$ period to a high degree of accuracy, and Snodgrass (1983) also finds his steep differential law to be time invariant (furthermore the $15 \mathrm{yr}$ period he used is covered by our $26 \mathrm{yr}$ period). Both analyses are based on $\mathrm{Mt}$ Wilson daily magnetograms (for the autocorrelation analysis supplemented by Kitt Peak magnetograms from 1976 onwards).

\section{Regeneration vs. Redistribution of Magnetic Flux}

The coexistence of these two entirely different rotation laws cannot be explained in terms of flux redistribution on the solar surface, which otherwise in principle could cause large differences between the plasma velocity and the pattern phase velocity. The most general and elaborate flux redistribution model is due to Sheeley et al. (1987). It shows, both analytically and in particular by numerical simulation, how a quasi-rigid pattern phase velocity can develop from a steeply differential plasma velocity by flux redistribution processes, like turbulent diffusion and meridional circulation. The reason why any such model completely fails to explain the coexistence of the two rotation laws is that if it is used to explain a quasi-rigid rotation law for the autocorrelation analysis, it also predicts practically the same quasi-rigid law if Snodgrass (1983) cross-correlation analysis is applied, in striking contrast with the steep latitude dependence that he found. This conclusion follows both from the numerical simulations and the analytical expressions of such a general redistribution model.

Snodgrass (1983) analyses the longitude variation of the magnetic field in daily magnetograms, and determines by cross-correlation how the field pattern is displaced in longitude 
through comparison of magnetograms separated in time by 1-4 days. His spatial resolution is about one min of arc. No individual flux features are identified, but the pattern itself is used as a tracer for the rotation. It is therefore surprising that the pattern phase velocity obtained agrees so closely with the plasma velocity obtained from Doppler measurements, in contradiction with redistribution models like that of Sheeley et al. (1987).

The autocorrelation analysis on the other hand has been based on synoptic data sampled at the central meridian in daily magnetograms. This results in a $26 \mathrm{yr}$ time series for each of the 30 latitude zones. As the sun rotates, the pattern recurs at the central meridian after an integer number of rotation periods, resulting in well-defined peaks in the autocorrelation functions at lags of an integer number of rotations. The precise lag at which these peaks occur gives the rotation period for the different latitudes. No significant dependence of the rotation period with peak number is found.

The basic difference between the cross-correlation and autocorrelation methods is thus the time scales involved. In the cross-correlation analysis lags of 1-4 days are used, in the autocorrelation analysis 27 days or more. The reason why flux redistribution models fail is that they implicitly assume that the magnetic flux that we see in magnetograms at any given time is dominated by old flux that has been around at the solar surface for a long time (many solar rotations), and is only shuffled around on the surface. In this picture it does not matter if the correlation analysis uses a lag of 27 days or 1 day; approximately the same pattern phase velocity of the old magnetic fluxes will be picked up in both cases.

To explain the simultaneous coexistence of the two rotation laws we are therefore forced to introduce the requirement that the magnetic-field pattern is regenerated over a time scale that is shorter than 27 days but longer than 4 days. Such an extremely short pattern turnover time is indicated by video magnetograph observations of flux emergence and cancellation rates (cf. Martin, 1989). The pattern that recurs at the central meridian after one or more solar rotations then does not consist of the same magnetic fluxes, but of new, recently emerged fluxes. This picture is reminiscent of the old concept of active longitudes. The rotation rate derived from the autocorrelation analysis is then not characteristic of the pattern phase velocity in the photosphere, but of the pattern phase velocity in the source region inside the sun, from which new flux is constantly being "emitted" to the surface.

\section{Time Invariance of the Quasi-rigid Rotation Law}

Next we want to identify the source region and constrain its properties by determining the possible time variations in the phase velocity of the synoptic magnetic field pattern. Such variations could arise due to the following causes:

- The depth distribution of the sources inside the sun varies with the solar cycle (and the angular velocity varies with depth).

- The phase velocity in the source region may differ from the plasma velocity there, and this difference, being determined by some dynamo wave, may be expected to vary with the solar cycle (the evolution of the dynamo).

- There may be torsional oscillations in the source region.

- The connection between the source and the emergence at the surface may not be strictly in the radial direction, but the path may be curved, connecting different latitudes. In this case the latitude migration of the sources may cause variations in the observed rotation rate.

- We may have overlooked some effect of flux redistribution of old flux at the surface. In this 
case, however, since it is the low-latitude active regions that would be the main "source" of the high-latitude flux, and since this "source" migrates in latitude and varies in amplitude with the cycle, time variations in the determined phase velocity would be expected.

As we will see below, however, our analysis shows that the rotation rate is constant, with very tight limits on the possible time variation. This severely constrains or partly rules out the above possibilities, and allows us to make rather far-reaching conclusions concerning the distribution of magnetic fields and angular velocity inside the sun.

To search for possible time variations in the phase velocity of the magnetic-field pattern, the $26 \mathrm{yr}$ time series has been divided into 21 shorter periods, each 16 Carrington periods $(16 \times 27.2753$ days $\approx 1.2 \mathrm{yr})$ long. As there are 30 latitude zones, there are $21 \times 30=630$ time series to analyse. For each time series a power spectrum has been computed. The sun's rotation shows up in the form of power spectrum peaks at frequencies that are an integer multiple $m$ of the rotation frequency (the inverse of the synodic rotation period). $m=1$ corresponds to a sine wave with a wavelength equal to the rotation period, $m=2$ to the second harmonic, etc. The frequency of each peak defines a rotation period of the pattern.

In Figure 2 we illustrate six of the 630 power spectra, for six different latitudes in the southern hemisphere and selected time periods (numbered from 1 to 21 ). The frequency is
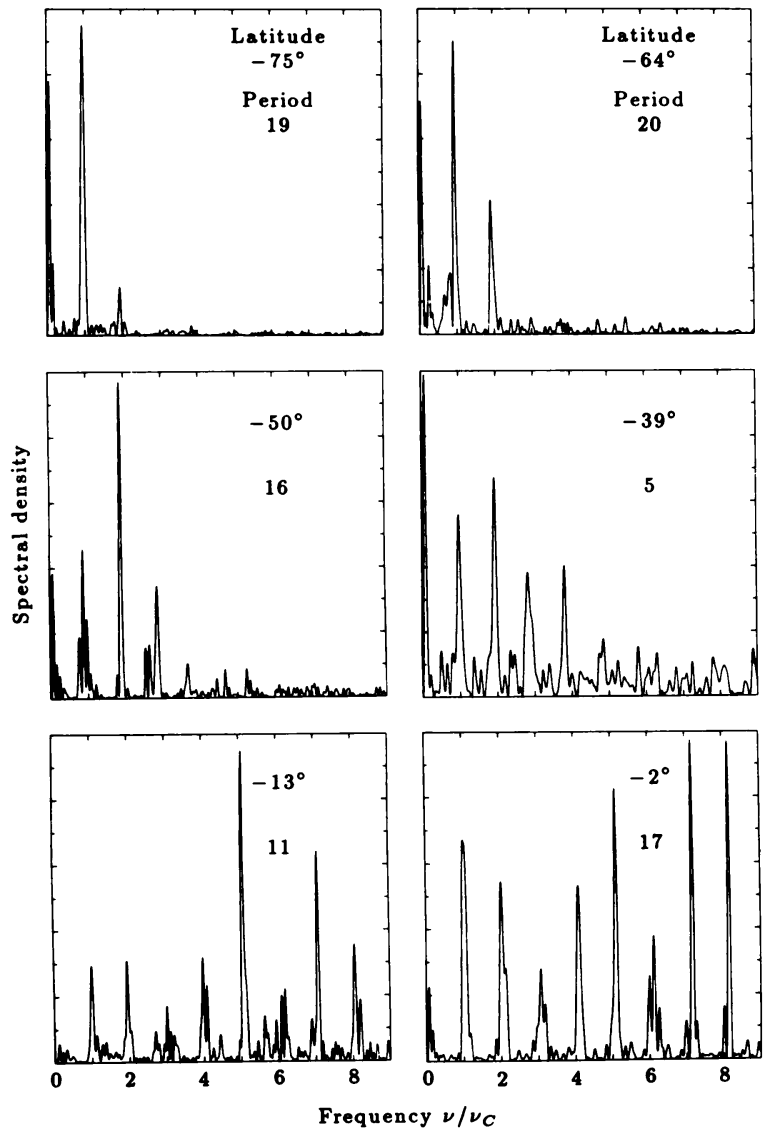

Fig. 2. Power spectra for selected latitude zones and time periods. $\nu_{C}$ is the Carrington frequency. 
given in units of the Carrington frequency $\left(1 / 27.2753\right.$ days $\left.^{-1}\right)$. Near the poles the peaks with the smallest $m$ numbers (representing the large-scale structures) dominate, while at lower latitudes the power is shifted to the higher harmonics (smaller scales).

The outcome of this analysis is that no significant variations of the rotation rate with the solar cycle is found, within the numerical error bars. For each power spectrum from which a well-defined rotation rate can be derived (the majority of the power spectra), the rotation rate is found to agree with the quasi-rigid rotation rate given by the global autocorrelation results. To suppress the apparently random fluctuations around this mean rotation rate to try to bring out any possible solar cycle variations, we have averaged the corresponding latitudes in the north and south hemispheres and applied a $3.7 \mathrm{yr}$ smoothing time window, to filter out the fluctuations with periods much shorter than the cycle period. No systematic, cycle-dependent pattern in the deviations $\Delta \omega$ from the mean sidereal angular velocity $\omega$ is found. Also "modal cleaning" (Stenflo, 1988) by harmonic decomposition of the unsmoothed $\Delta \omega$ pattern fails to reveal an underlying solar-cycle pattern.
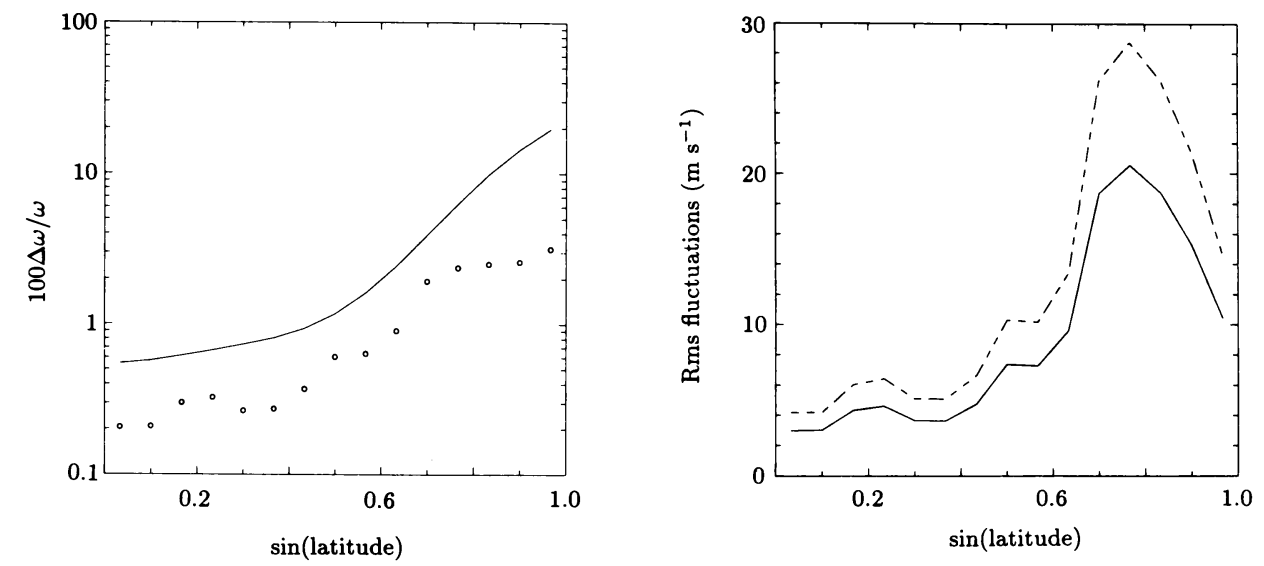

Fig. 3. Open circles in the left diagram: Rms fluctuations of the angular velocity $\omega$ around the mean value (the quasi-rigid rotation law), expressed in percent. Right diagram: The rms fluctuations converted to linear velocity $\left(\mathrm{m} \mathrm{s}^{-1}\right)$, assuming that the observed pattern phase velocity refers to the bottom (solid line) or the top (dashed line) of the convection zone. Left diagram, solid line: The difference between the angular velocities of Snodgrass (1983) and the autocorrelation results, expressed in percent.

Figure 3 shows that the rms fluctuations of the smoothed $\omega$ values are $\lesssim 0.3 \%$, or $\lesssim 4$ $\mathrm{m} \mathrm{s}^{-1}$ at lower latitudes, but increases to $2-3 \%\left(10-20 \mathrm{~m} \mathrm{~s}^{-1}\right)$ at higher latitudes (where the amount of magnetic flux and thus the signal-to-noise ratio is smaller). While these numbers represent rms fluctuations around a mean level, the systematic deviation of the Snodgrass curve reaches as much as $20 \%$ at the poles.

Our values for the rms fluctuations can be regarded as one-sigma upper limits to possible solar-cycle variations of the pattern phase velocity of the sources of magnetic flux in the solar interior. These very low upper limits suggest that the depth distribution of the sources does not vary much with the solar cycle, otherwise the depth variation of the angular velocity would show up as a time variation in the pattern phase velocity. This speaks in favour of 
flux storage at the bottom of the convection zone with the dynamo operating there, rather than being distributed within the convection zone, in agreement with theoretical arguments by a number of authors (e.g. Spiegel and Weiss, 1980; DeLuca, 1987; Schüssler, 1987).

The absence of significant time variations also seems to favour the view that the pattern phase velocity closely represents the plasma velocity in the source region, since one would expect a difference generated by a dynamo wave to vary with the phase of the solar cycle, which does not seem to be the case. Our upper limits are also similar in magnitude or smaller than the amplitudes of the "torsional oscillations" of the observed surface Doppler velocities (Howard and LaBonte, 1980; LaBonte and Howard, 1982). Torsional oscillations in the source region (at the bottom of the convection zone), if they exist at all, should therefore be smaller than these limits.

There are thus good reasons why the quasi-rigid rotation law should represent the plasma velocity near the base of the convection zone, while the Snodgrass law represents the plasma velocity at the top of the convection zone. This appears to be consistent with the results of helioseismology. Interpolating between the top and bottom of the convection zone we obtain isocontours for the angular velocity which are very similar to the theoretically derived contours in Fig. 2 of Rüdiger and Tuominen (1989).

\section{References}

DeLuca, E.E. (1987) 'Dynamo theory for the interface between the convection zone and the radiative interior of a star', NCAR Cooperative Thesis No. 104, Boulder, Colorado.

Howard, R. and LaBonte, B. (1980) 'The Sun is observed to be a torsional oscillator with a period of 11 years', Astrophys. J. 239, L33-L36.

Howard, R., Adkins, J.M., Boyden, J.E., Cragg, T.A., Gregory, T.S., LaBonte, B.J., Padilla, S.P., and Webster, L. (1983) 'Solar rotation results at Mount Wilson', Solar Phys. 83, 321-338.

LaBonte, B.J. and Howard, R. (1982) 'Torsional waves on the sun and the activity cycle', Solar Phys. 75, 161-178.

Martin, S.F. (1989) 'Evolution of small-scale magnetic fields', in these proceedings.

Rüdiger, G. and Tuominen, I. (1989) 'Generators of solar differential rotation and implications of helioseismology', in these proceedings.

Schüssler, M. (1987) 'Magnetic fields and the rotation of the solar convection zone', in B.R. Durney and S. Sofia (eds.), The Internal Solar Angular Velocity, Astrophys. Space Sci. Library 137, 303-320.

Sheeley, N.R., Jr., Nash, A.G., and Wang, Y.-M. (1987) 'The origin of rigidly rotating magnetic field patterns on the sun', Astrophys. J. 319, 481-502.

Snodgrass, H.B. (1983) 'Magnetic rotation of the solar photosphere', Astrophys. J. 270, 288-299.

Spiegel, E.A. and Weiss, N.O. (1980) 'Magnetic activity and variations in solar luminosity', Nature 287, 616-617.

Stenflo, J.O. (1988) 'Global wave patterns in the Sun's magnetic field', Astrophys. Space Sci. $144,321-336$.

Stenflo, J.O. (1989) 'Differential rotation of the Sun's magnetic field pattern', Astron. Astrophys. 210, 403-409.

Van Tend, W. and Zwaan, C. (1976) 'On differences in differential rotation', in V. Bumba and J. Kleczek (eds.), Basic Mechanisms of Solar Activity, IAU Symp. 71, 45-46. 